\title{
Mirogabalin vs pregabalin for chemotherapy-induced peripheral neuropathy in pancreatic cancer patients
}

\author{
Mitsuru Sugimoto ${ }^{1 *}$, Tadayuki Takagi ${ }^{1}$, Rei Suzuki ${ }^{1}$, Naoki Konno ${ }^{1}$, Hiroyuki Asama' , Yuki Sato ${ }^{1}$, Hiroki Irie , \\ Yoshinori Okubo 1,2, Jun Nakamura, ${ }^{1,2}$, Mika Takasumi ${ }^{1}$, Minami Hashimoto ${ }^{1,2}$, Tsunetaka Kato 1,2, \\ Ryoichiro Kobashi ${ }^{1}$, Takuto Hikichi ${ }^{2}$ and Hiromasa Ohira ${ }^{1}$
}

\begin{abstract}
Background: The prognosis of pancreatic cancer (PC) has been improved by new chemotherapy regimens (combination of 5-fluorouracil, oxaliplatin, irinotecan, and leucovorin (FOLFIRINOX) or gemcitabine plus nab-paclitaxel (GnP)). Unfortunately, chemotherapy-induced peripheral neuropathy (CIPN) is a common adverse event of these two regimens. The efficacy of pregabalin for CIPN has been reported in previous studies. However, the efficacy of mirogabalin for CIPN remains unknown. Thus, in this study, we aimed to clarify which drug (mirogabalin or pregabalin) was more valuable for improving CIPN.
\end{abstract}

Methods: A total of 163 PC patients who underwent FOLFIRINOX or GnP between May 2014 and January 2021 were enrolled. Among them, 34 patients were diagnosed with CIPN. Thirteen patients were treated with mirogabalin (mirogabalin group), and twenty-one patients were treated with pregabalin (pregabalin group). Treatment efficacy was compared between the two groups.

Results: In both the mirogabalin group and the pregabalin group, the grade of patients with CIPN at 2, 4, and 6 weeks after the initiation of treatment showed significant improvement compared to the pretreatment grade. Notably, the rate of CIPN improvement was higher in the mirogabalin group than in the pregabalin group (2 weeks: $84.6 \%$ $(11 / 13)$ vs $33.3 \%(7 / 21), P$ value $=0.005 ; 4$ weeks, 6 weeks: $92.3 \%(12 / 13)$ vs $33.3 \%(7 / 21), P$ value $=0.001)$

Conclusions: Although both mirogabalin and pregabalin were effective at improving CIPN, mirogabalin might be a suitable first choice for CIPN in PC patients.

Trial registration: Not applicable

Keywords: chemotherapy-induced peripheral neuropathy, mirogabalin, pregabalin, pancreatic cancer

\section{Background}

Pancreatic cancer (PC) is a lethal disease that has become a major cause of cancer-related death worldwide [1-3]. The poor prognosis of most PC patients is due to the

\footnotetext{
*Correspondence: kita335@fmu.ac.jp

${ }^{1}$ Department of Gastroenterology, School of Medicine, Fukushima

Medical University, Fukushima, Japan

Full list of author information is available at the end of the article
}

advanced stage of the disease at diagnosis, making resection difficult [4-6]. Thus, chemotherapy has become the general treatment strategy for PC patients. Recently, new chemotherapy regimens have been developed, such as the combination of 5-fluorouracil, oxaliplatin, irinotecan, and leucovorin (FOLFIRINOX) or gemcitabine plus nabpaclitaxel (GnP). Although the prognosis of PC patients is very poor, it has been dramatically improved by FOLFIRINOX or GnP [7-35]. On the other hand, many 
adverse events are also reported for these regimens. Chemotherapy-induced peripheral neuropathy (CIPN) is a common side effect of both FOLFIRINOX and GnP. In past reports, the frequency of grade 3-4 CIPN was 0 - 25\% for FOLFIRINOX $[7-9,11,12,14,15,17,18,21-$ $23,36]$ and $1.8-30.4 \%$ for GnP [27-31, 35, 37]. Oxaliplatin and paclitaxel represent a class of neurotoxic drugs [38-40]. When CIPN becomes severe, it could influence the decision to continue chemotherapy, affecting patient prognosis. Therefore, adequate management of CIPN is necessary.

Regarding drug treatments for CIPN, the efficacy of duroxetine was demonstrated in a past large double-blind randomized controlled trial [41]. In addition, pregabalin was found to be more valuable for treating CIPN than duroxetine in some reports $[42,43]$. On the other hand, the efficacy of mirogabalin for diabetic peripheral neuropathy has also been reported [44]. Recently, mirogabalin treatment for CIPN was covered by medical insurance in Japan. Unfortunately, the efficacy of mirogabalin for CIPN is unknown. Therefore, in this study, we compared mirogabalin and pregabalin for the treatment of CIPN.

\section{Methods}

\section{Study design and ethics}

This was a retrospective study comparing the efficacy of mirogabalin and pregabalin for the treatment of CIPN. This study was approved by the Institutional Review Board of Fukushima Medical University (approval number: 29254). The analysis used anonymous clinical data obtained after all the participants agreed to treatment by written consent, so patients were not required to give informed consent for the study. Informed consent was obtained from all participants or, if participants were under 18, from a parent and/or legal guardian. The details of the study can be found on the homepage of Fukushima Medical University. All methods were carried out in accordance with relevant guidelines and regulations.

\section{Patients}

A total of 163 PC patients who underwent FOFIRINOX or GnP therapy at Fukushima Medical University between May 2014 and January 2021 were enrolled. Among them, 34 patients were diagnosed with CIPN based on its clinical course. When a PC patient who was administered a neurotoxic drug reported new pain or numbness on the extremities, the patient was diagnosed with CIPN [45]. Thirteen patients were treated with mirogabalin (mirogabalin group), and twenty-one patients were treated with pregabalin (pregabalin group) (Fig. 1). PC was diagnosed by endoscopic ultrasonography-guided fine needle aspiration, abdominal ultrasonography-guided biopsy, bile cytology, or biliary biopsy.

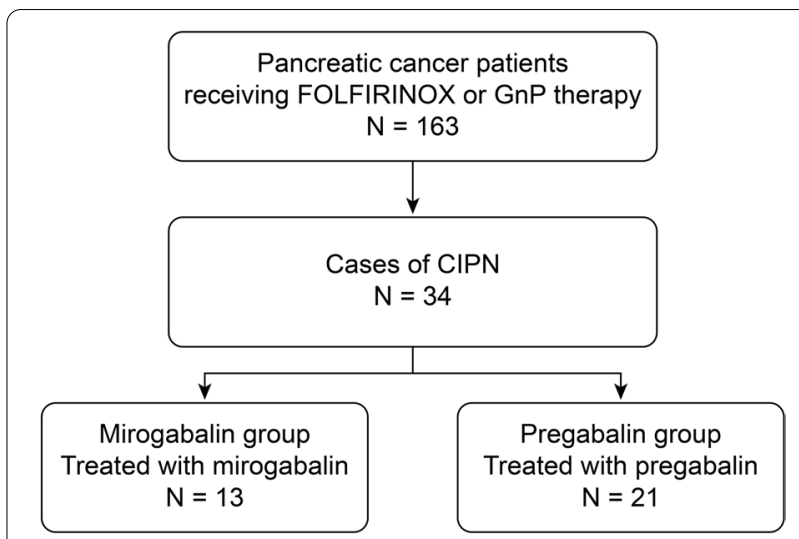

Fig. 1 Flowchart of patient selection

\section{Dose of mirogabalin, pregabalin}

The dosages of mirogabalin and pregabalin were determined by each doctor, and the effect of mirogabalin or pregabalin was evaluated every one or two weeks. If CIPN did not improve, the dose of mirogabalin or pregabalin was increased. On the other hand, when a side effect of mirogabalin or pregabalin was observed, the dose was decreased. When a side effect became severe or CIPN was sufficiently improved, mirogabalin or pregabalin was stopped. The actual dosage of mirogabalin was as follows (10 mg/day for 4-6 weeks: six patients, $10 \mathrm{mg} /$ day for a week $\rightarrow 20 \mathrm{mg} /$ day for a week $\rightarrow 30 \mathrm{mg} /$ day for four weeks: a patient, $10 \mathrm{mg} /$ day for a week $\rightarrow 15 \mathrm{mg} /$ day for a week $\rightarrow 20 \mathrm{mg} /$ day for four weeks: a patient, $5 \mathrm{mg} /$ day for a week $\rightarrow 15 \mathrm{mg} /$ day for two weeks $\rightarrow 10 \mathrm{mg} /$ day for a week $\rightarrow 20 \mathrm{mg} /$ day for two weeks: a patient, $10 \mathrm{mg} /$ day for two weeks $\rightarrow 20 \mathrm{mg} /$ day for four weeks: a patient, $10 \mathrm{mg} /$ day for four weeks $\rightarrow 20 \mathrm{mg} /$ day for two weeks: a patient, $10 \mathrm{mg} /$ day for a week $\rightarrow 20 \mathrm{mg} /$ day for five weeks: a patient, $5 \mathrm{mg} /$ day for two weeks $\rightarrow 10 \mathrm{mg} /$ day for four weeks: a patient). The actual dosage of pregabalin was as follows $(150 \mathrm{mg} /$ day for 2-6 weeks: 18 patients, $75 \mathrm{mg} /$ day for 6 weeks: a patient, $150 \mathrm{mg} /$ day for three weeks $\rightarrow 75 \mathrm{mg} /$ day for three weeks: a patient, $150 \mathrm{mg} /$ day for four weeks $\rightarrow 75 \mathrm{mg}$ for two weeks: a patient).

\section{Examination items}

Patient characteristics and background (age, sex, tumor stage based on the Union for International Cancer Control (UICC) classification $8^{\text {th }}$ edition [46], neurotoxic regimen, concomitant drugs for CIPN, pretreatment CIPN grade) were compared between the mirogabalin group and the pregabalin group. CIPN grade was compared between pretreatment and at 2 weeks after treatment, 4 weeks after treatment, or 6 weeks after treatment. The 
grade of CIPN was classified by Common Terminology Criteria for Adverse Events (CTCAE) version 5.0. The number of patients with improvement in CIPN at 2, 4, or 6 weeks after treatment was compared between the mirogabalin group and the pregabalin group. If a patient stopped taking medicine for several reasons (CIPN was sufficiently improved, drugs were ineffective, adverse events), the evaluation of improvement in CIPN was continued until 6 weeks after drug initiation.

\section{Statistical analysis}

Continuous variables following a normal distribution (age) were analyzed with Welch's t-test. Ordinal variables and continuous variables that did not follow a normal distribution were analyzed with the Mann-Whitney U test. Nominal variables were analyzed with Fisher's exact test. The treatment effect of each group was compared between pretreatment and posttreatment with the Wilcoxon signed-rank test. $P<0.05$ was defined as statistically significant. All statistical analyses were performed using EzR (Saitama Medical Centre, Jichi Medical University, Saitama, Japan).

\section{Results}

\section{Patient characteristics and clinical background}

Age, sex, UICC stage, neurotoxic regimen, and concomitant drugs for CIPN were not different between the two groups (Table 1). In both groups, the majority of patients underwent GnP. The pretreatment CIPN grade was significantly higher in the mirogabalin group than in the pregabalin group $(3(2-3)$ vs $2(2-3), P<0.01)$.

\section{Treatment effect for CIPN}

CIPN showed improvement in both the mirogabalin group and pregabalin group (Figure 2). In each group, the grade of CIPN at 2 weeks, 4 weeks, and 6 weeks after drug initiation showed significant improvement over the pretreatment grade.

The rate of improvement in CIPN at 2, 4 or 6 weeks after drug initiation was significantly higher in the mirogabalin group than in the pregabalin group (2 weeks: $84.6 \%(11 / 13)$ vs $33.3 \%(7 / 21), P$ value $=0.005 ; 4$ weeks, 6 weeks: $92.3 \%(12 / 13)$ vs $33.3 \%(7 / 21), P$ value $=0.001)$ (Fig. 3).

\section{Drug discontinuation and adverse events}

Drug discontinuation are shown in Table 2. Mirogabalin was stopped in two (15.4\%) patients 4 weeks after it was initiated; one patient reported dizziness, and CIPN was found to improve very well in the other patient. Pregabalin was stopped in eleven (52.4\%) patients. Seven (33.3\%) patients stopped taking pregabalin 2 weeks after pregabalin was initiated. Sufficient improvement in CIPN was not observed in two patients, and side effects were observed in the other five patients. Four (19.0\%) patients stopped taking pregabalin four weeks after pregabalin was initiated.

Table 1 Comparison of patient characteristics and clinical background

\begin{tabular}{|c|c|c|c|}
\hline & $\begin{array}{l}\text { Mirogabalin group } \\
(N=13)\end{array}$ & $\begin{array}{l}\text { Pregabalin group } \\
(N=21)\end{array}$ & $P$ value \\
\hline Age, years & $61.3 \pm 13.6$ & $65.4 \pm 7.2$ & 0.33 \\
\hline Sex, male/female & $8 / 5$ & $9 / 12$ & 0.48 \\
\hline UICC stage, median (range) & $4(3-4)$ & $4(2-4)$ & 0.74 \\
\hline$\|, n(\%)$ & $0(0)$ & $1(4.8)$ & \\
\hline III, n (\%) & $5(38.5)$ & $8(38.1)$ & \\
\hline $\mathrm{IV}, \mathrm{n}(\%)$ & $8(61.5)$ & 12(57.1) & \\
\hline Neurotoxic regimen, n (\%) & & & 0.68 \\
\hline FOLFIRINOX & $2(15.4)$ & $5(23.8)$ & \\
\hline $\mathrm{GnP}$ & $11(84.6)$ & $16(76.2)$ & \\
\hline Concomitant drugs for CIPN, n (\%) & $3(23.1)$ & $5(23.8)$ & 1.0 \\
\hline Duroxetine & $1(7.7)$ & $1(4.8)$ & \\
\hline Vitamin B12 & $2(15.4)$ & $1(4.8)$ & \\
\hline Duroxetine, goshajinkigan & & $1(4.8)$ & \\
\hline Goshajinkigan & & $2(9.5)$ & \\
\hline Pretreatment CIPN grade, median (range) & $3(2-3)$ & $2(2-3)$ & $<0.01$ \\
\hline $2, n(\%)$ & $1(7.7)$ & $13(61.9)$ & \\
\hline $3, \mathrm{n}(\%)$ & $12(92.3)$ & $8(38.1)$ & \\
\hline
\end{tabular}

Values are shown as the mean \pm standard deviation, median (range) or $\mathrm{n}(\%)$

UICC Union for International Cancer Control classification; FOLFIRINOX combination of 5-fluorouracil, oxaliplatin, irinotecan, and leucovorin; GnP gemcitabine plus nab-paclitaxel; CIPN chemotherapy-induced peripheral neuropathy 


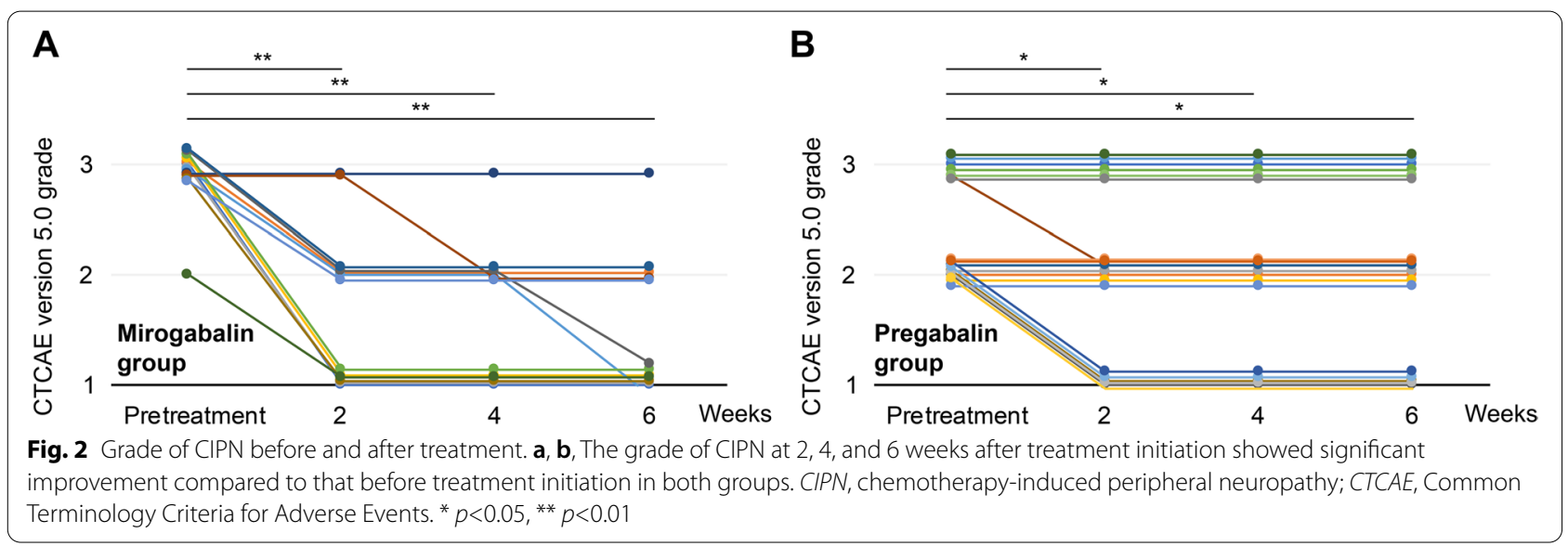

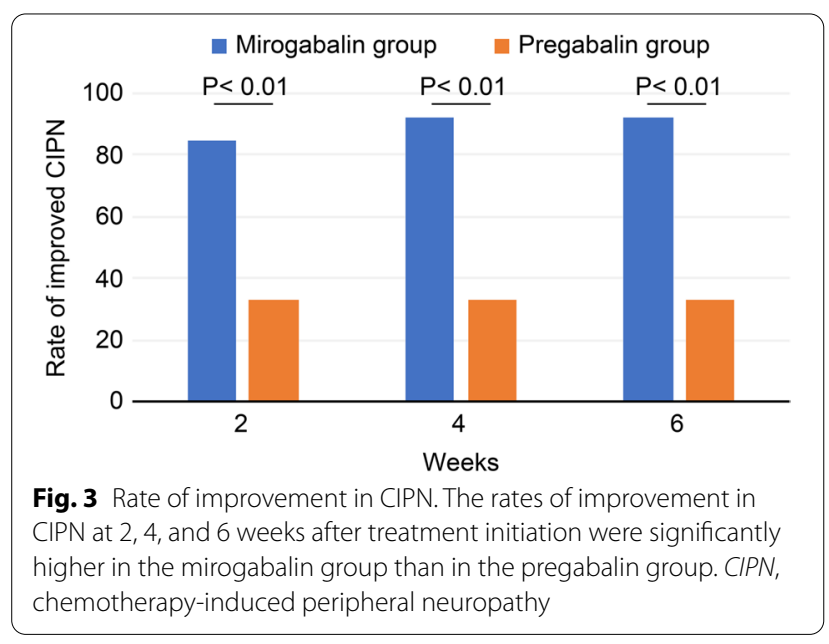

Table 2 The reasons for drug discontinuation

\begin{tabular}{|c|c|c|}
\hline $\begin{array}{l}\text { Reason for drug } \\
\text { discontinuation, } \mathrm{n}(\%)\end{array}$ & $\begin{array}{l}\text { Mirogabalin } \\
\text { group } \\
(N=13)\end{array}$ & $\begin{array}{l}\text { Pregabalin group } \\
(N=21)\end{array}$ \\
\hline 2 weeks after initiation & $0(0)$ & $7(33.3)$ \\
\hline Ineffective & $0(0)$ & $2(9.5)$ \\
\hline Adverse events & $0(0)$ & $5(23.8)$ \\
\hline 4 weeks after initiation & $2(15.4)$ & $4(19.0)$ \\
\hline CIPN sufficiently improved & $1(7.7)$ & $1(4.8)$ \\
\hline Adverse events & $1(7.7)$ & $0(0)$ \\
\hline Ineffective & $0(0)$ & $3(14.3)$ \\
\hline
\end{tabular}

Values are shown as $\mathrm{n}(\%)$

CIPN, chemotherapy-induced peripheral neuropathy

Sufficient improvement in CIPN was not observed in three patients, and CIPN was found to improve very well in the other patient. The change in CTCAE grade was not observed after drug discontinuation.
Table 3 The comparison of adverse events

\begin{tabular}{|c|c|c|c|}
\hline & $\begin{array}{l}\text { Mirogabalin } \\
\text { group } \\
(N=13)\end{array}$ & $\begin{array}{l}\text { Pregabalin group } \\
(N=21)\end{array}$ & $P$ value \\
\hline Adverse events, n (\%) & $2(15.4)$ & $7(33.3)$ & 0.43 \\
\hline Dizziness & $1(7.7)$ & $2(9.5)$ & \\
\hline Edema & $1(7.7)$ & $2(9.5)$ & \\
\hline Sleepiness & 0 & $3(14.3)$ & \\
\hline
\end{tabular}

Values are shown as $\mathrm{n}(\%)$

Adverse events were not significantly different between the mirogabalin group and the pregabalin group (Table 3 ).

\section{Discussion}

In this study, the majority of CIPN occurred by GnP. The therapeutic effect for CIPN was compared between mirogabalin and pregabalin. Both drugs were effective at improving CIPN in PC patients. Although the effect of mirogabalin on CIPN was unknown, the rate of improved CIPN was significantly higher in the mirogabalin group than in the pregabalin group.

In a recent meta-analysis that compared treatment outcomes between FOLFIRINOX and GnP, CIPN occurred much more frequently in patients who were treated with $\mathrm{GnP}$ than in patients who were treated with FOLFIRINOX [47]. Therefore, it was reasonable that the regimen responsible for most CIPN was GnP in this study.

Several drugs have been reported for treating CIPN. In these reports, calcium and magnesium, goshajinkigan, duloxetine, vitamin B12, pregabalin, and gabapentin were used [41-43, 48-62]. However, calcium/magnesium, goshajinkigan, and gabapentin were found to be ineffective at treating CIPN in the largest double-blind randomized controlled trials (RCTs) for each drug. Vitamin B12 was used as a control group in the study to investigate the 
efficacy of goshajinkigan and duroxetine. In the largest RCT, duroxetine was found to be effective at treating CIPN. In addition, the efficacy of pregabalin for CIPN was reported to be better than that of duroxetine in two reports. In 2018, Avan et al. [42] performed a doubleblind RCT that targeted 82 breast cancer patients with taxane-induced peripheral neuropathy (pregabalin group: $\mathrm{n}=40$, duroxetine group: $\mathrm{n}=42$ ). In their study, pregabalin provided the greatest improvement in insomnia and pain scores [42]. In 2019, Salehifar et al. [43] reported that pregabalin was more valuable for improving the sensory and pain scores of CIPN than duroxetine. In both reports, CIPN was improved after 6 weeks of pregabalin treatment. In this study, CIPN was significantly improved after pregabalin treatment. Although mirogabalin was reported to be useful for diabetic neuropathy [44], it was also found to be useful for CIPN in this study.

Although mirogabalin and pregabalin were both valuable for improving CIPN, the treatment effect was different between the two groups. Although no significant difference in adverse events was observed, adverse events were more common in the pregabalin group than in the mirogabalin group. Pregabalin and mirogabalin combine with the $\alpha 2 \delta$ subunit of Ca channels in the back horn of the spinal cord and impede the inflow of calcium, which is required for neurotransmitter release $[44,63-66]$. However, the two drugs show different connectivity to the subtypes of the $\alpha 2 \delta$ subunit of the $\mathrm{Ca}$ channel. Among these subtypes, the $\alpha 2 \delta-1$ subunit is related to analgesic effects [67], and the $\alpha 2 \delta-2$ subunit is related to central nervous system disorders [68]. In a past report written by Domon et al. [69], the dissociation half-life between mirogabalin and the $\alpha 2 \delta-1$ subunit was 11.1 (8.3-16.4) hours, and the dissociation half-life between mirogabalin and the $\alpha 2 \delta-2$ subunit was 2.4 (2.12.8 ) hours. On the other hand, the dissociation half-life between pregabalin and $\alpha 2 \delta-1$ and $\alpha 2 \delta-2$ subunits was 1.4 hours $(\alpha 2 \delta-1$ : $1.4(1.3-1.4)$ hours, $\alpha 2 \delta$-2: 1.4 (0.9-2.7) hours) [69]. Because the dissociation half-life between mirogabalin and the $\alpha 2 \delta-1$ subunit was longer than that between mirogabalin and the $\alpha 2 \delta-2$ subunit, the analgesic effect is expected to be durable, and adverse events caused by central nervous system disorder are expected to be reduced by mirogabalin. This difference in the connection to the $\alpha 2 \delta$ subunit leads to differences in not only the treatment effects but also the adverse events elicited by mirogabalin and pregabalin.

There were some limitations to this study that should be mentioned. First, this was a retrospective study with a small sample size conducted at a single institution. However, this study is the first to compare the efficacy of mirogabalin and pregabalin for the treatment of CIPN. We hope that multicenter RCTs will be conducted in the future to confirm the results reported in this study. Second, the doses of mirogabalin and pregabalin were not uniform. The results showed that both drugs were effective at treating CIPN, even though a low dose was used for both drugs.

\section{Conclusions}

Although both mirogabalin and pregabalin were effective at improving CIPN, a higher rate of improved CIPN was observed in patients who were treated with mirogabalin. Mirogabalin might be a suitable first choice for CIPN in PC patients.

\section{Abbreviations}

PC: Pancreatic cancer; FOLFIRINOX: The combination of 5-fluorouracil, oxaliplatin, irinotecan, and leucovorin; GnP: Gemcitabine plus nab-paclitaxel; CIPN: Chemotherapy-induced peripheral neuropathy.

\section{Acknowledgements}

We thank all the staff at the Department of Gastroenterology of Fukushima Medical University, the Department of Endoscopy of Fukushima Medical University Hospital, and the gastroenterology ward of Fukushima Medical University Hospital. We also thank American Journal Experts for providing English language editing.

\section{Informed consent}

The analysis used anonymous clinical data obtained after all the participants agreed to treatment by written consent, so patients were not required to give informed consent for the study. Informed consent was obtained from all participants or, if participants were under 18 , from a parent and/or legal guardian.

\section{Authors' contributions}

MS wrote the paper and designed and performed the research; TT designed and oversaw the research; RS, NK, HA, YS, HI, YO, JN, MT, MH, TK, RK, and TH provided clinical advice; and $\mathrm{HO}$ supervised the report and the writing of the paper. All authors have read and approved the final manuscript.

\section{Funding}

Department of Gastroenterology, Fukushima Medical University

\section{Availability of data and materials}

The datasets generated and/or analyzed during the current study are available from the corresponding author upon reasonable request.

\section{Declarations}

\section{Ethics approval and consent to participate}

The study protocol was reviewed and approved by the Institutional Review Board of Fukushima Medical University (Number 29254). The analysis used anonymous clinical data obtained after all the participants agreed to treatment by written consent, so patients were not required to give informed consent for the study. The details of the study can be found on the homepage of Fukushima Medical University.

\section{Consent for publication}

Not applicable

\section{Competing interests}

The authors declare that they have no competing interests to report.

\section{Author details}

${ }^{1}$ Department of Gastroenterology, School of Medicine, Fukushima Medical University, Fukushima, Japan. ${ }^{2}$ Department of Endoscopy, Fukushima Medical University Hospital, Fukushima, Japan. 
Received: 23 March 2021 Accepted: 26 November 2021

Published online: 09 December 2021

\section{References}

1. Bray F, Ferlay J, Soerjomataram I, Siegel RL, Torre LA, Jemal A. Global cancer statistics 2018: GLOBOCAN estimates of incidence and mortality worldwide for 36 cancers in 185 countries. CA Cancer J Clin. 2018;68:394-424.

2. Siegel RL, Miller KD, Jemal A. Cancer statistics, 2020. CA Cancer J Clin. 2020;70:7-30.

3. Afshar N, English DR, Chamberlain JA, Blakely T, Thursfield V, Farrugia $H$, et al. Differences in cancer survival by remoteness of residence: an analysis of data from a population-based cancer registry. Cancer Causes Control. 2020;31:617-29.

4. Stathis A, Moore MJ. Advanced pancreatic carcinoma: current treatment and future challenges. Nat Rev Clin Oncol. 2010;7:163-72.

5. Warsame R, Grothey A. Treatment options for advanced pancreatic cancer: a review. Expert Rev Anticancer Ther. 2012;12:1327-36.

6. Fogel EL, Shahda S, Sandrasegaran K, DeWitt J, Easler JJ, Agarwal DM, et al. A multidisciplinary approach to pancreas cancer in 2016: a review. Am J Gastroenterol. 2017;112:537-54.

7. Conroy T, Paillot B, François E, Bugat R, Jacob JH, Stein U, et al. Irinotecan plus oxaliplatin and leucovorin-modulated fluorouracil in advanced pancreatic cancer--a Groupe Tumeurs Digestives of the Federation Nationale des centres De Lutte Contre le Cancer study. J Clin Oncol. 2005;23:1228-36.

8. Conroy T, Desseigne F, Ychou M, Bouché O, Guimbaud R, Bécouarn Y, et al. FOLFIRINOX versus gemcitabine for metastatic pancreatic cancer. N Engl J Med. 2011:364:1817-25.

9. Assaf E, Verlinde-Carvalho M, Delbaldo C, Grenier J, Sellam Z, Pouessel D, et al. 5-fluorouracil/leucovorin combined with irinotecan and oxaliplatin (FOLFIRINOX) as second-line chemotherapy in patients with metastatic pancreatic adenocarcinoma. Oncology. 2011:80:301-6.

10. Peddi PF, Lubner S, McWilliams R, Tan BR, Picus J, Sorscher SM, et al. Multiinstitutional experience with FOLFIRINOX in pancreatic adenocarcinoma. JOP. 2012;13:497-501

11. Hosein PJ, Macintyre J, Kawamura C, Maldonado JC, Ernani V, LoaizaBonilla $A$, et al. A retrospective study of neoadjuvant FOLFIRINOX in unresectable or borderline-resectable locally advanced pancreatic adenocarcinoma. BMC Cancer. 2012:12:199.

12. Lorgis V, Chauffert B, Gentil J, Ghiringhelli F. Influcence of localization of primary tumor on effectiveness of 5-fluorouracil/leucovorin combined with irinotecan and oxaliplatin (FOLFIRINOX) in patients with metastatic pancreatic adenocarcinoma: a retrospective study. Anticancer Res. 2012;32:4125-30

13. Mahaseth H, Brutcher E, Kauh J, Hawk N, Kim S, Chen Z, et al. Modified FOLFIRINOX regimen with improved safety and maintained efficacy in pancreatic adenocarcinoma. Pancreas. 2013:42:1311-5.

14. Faris JE, Blaszkowsky LS, McDermott S, Guimaraes AR, Szymonifka J, Huynh MA, et al. FOLFIRINOX in locally advanced pancreatic cancer: the Massachusetts General Hospital Cancer Center experience. Oncologist. 2013;18:543-8.

15. Gunturu KS, Yao X, Cong X, Thumar JR, Hochster HS, Stein SM, et al. FOLFIRINOX for locally advanced and metastatic pancreatic cancer: single institution retrospective review of efficacy and toxicity. Med Oncol. 2013;30:361.

16. Hohla F, Hopfinger G, Romeder F, Rinnerthaler G, Bezan A, Stättner S, et al. Female gender may predict response to FOLFIRINOX in patients with unresectable pancreatic cancer: a single institution retrospective review. Int J Oncol. 2014;44:319-26.

17. Moorcraft SY, Khan K, Peckitt C, Watkins D, Rao S, Cunningham D, et al. FOLFIRINOX for locally advanced or metastatic pancreatic ductal adenocarcinoma: the Royal Marsden experience. Clin Colorectal Cancer 2014;13:232-8.

18. Marthey L, Sa-Cunha A, Blanc JF, Gauthier M, Cueff A, Francois E, et al. FOLFIRINOX for locally advanced pancreatic adenocarcinoma: results of an AGEO multicenter prospective observational cohort. Ann Surg Oncol. 2015;22:295-301.

19. Mellon EA, Hoffe SE, Springett GM, Frakes JM, Strom TJ, Hodul PJ, et al. Long-term outcomes of induction chemotherapy and neoadjuvant stereotactic body radiotherapy for borderline resectable and locally advanced pancreatic adenocarcinoma. Acta Oncol. 2015;54:979-85.

20. Sadot E, Doussot A, O'Reilly EM, Lowery MA, Goodman KA, Do RK, et al. FOLFIRINOX induction therapy for stage 3 pancreatic adenocarcinoma. Ann Surg Oncol. 2015;22:3512-21.

21. Zahir MN, Jabbar AA. Metastatic pancreatic carcinoma and experience with FOLFIRINOX - a cross sectional analysis from a developing country. Asian Pac J Cancer Prev. 2015;16:6001-6.

22. Rombouts SJ, Mungroop TH, Heilmann MN, van Laarhoven HW, Busch OR, Molenaar IQ, et al. FOLFIRINOX in locally advanced and metastatic pancreatic cancer: a single centre cohort study. J Cancer. 2016;7:1861-6.

23. Chllamma MK, Cook N, Dhani NC, Giby K, Dodd A, Wang L, et al. FOLFIRINOX for advanced pancreatic cancer: the Princess Margaret Cancer Centre experience. Br J Cancer. 2016;115:649-54.

24. Orlandi A, Calegari MA, Martini M, Cocomazzi A, Bagalà C, Indellicati G, et al. Gemcitabine versus FOLFIRINOX in patients with advanced pancreatic adenocarcinoma hENT1-positive: everything was not too bad back when everything seemed worse. Clin Transl Oncol. 2016;18:988-95.

25. Hann A, Bohle W, Egger J, Zoller WG. Improvement in advanced pancreatic cancer survival with novel chemotherapeutic strategies - experience of a community based hospital. Z Gastroenterol. 2016;54:1138-42.

26. Von Hoff DD, Ervin T, Arena FP, Chiorean EG, Infante J, Moore M, et al. Increased survival in pancreatic cancer with nab-paclitaxel plus gemcitabine. N Engl J Med. 2013;369:1691-703.

27. Zhang DS, Wang DS, Wang ZQ, Wang FH, Luo HY, Qiu MZ, et al. Phase I/ Il study of albumin-bound nab-paclitaxel plus gemcitabine administered to Chinese patients with advanced pancreatic cancer. Cancer Chemother Pharmacol. 2013;71:1065-72.

28. Goldstein D, El-Maraghi RH, Hammel P, Heinemann V, Kunzmann V, Sastre J, et al. nab-paclitaxel plus gemcitabine for metastatic pancreatic cancer: long-term survival from a phase III trial. J Natl Cancer Inst. 2015;107:dju413.

29. Ueno H, Ikeda M, Ueno M, Mizuno N, loka T, Omuro Y, et al. Phase I/ Il study of nab-paclitaxel plus gemcitabine for chemotherapy-naive Japanese patients with metastatic pancreatic cancer. Cancer Chemother Pharmacol. 2016:77:595-603.

30. Ahn DH, Krishna K, Blazer M, Reardon J, Wei L, Wu C, et al. A modified regimen of biweekly gemcitabine and nab-paclitaxel in patients with metastatic pancreatic cancer is both tolerable and effective: a retrospective analysis. Ther Adv Med Oncol. 2017;9:75-82.

31. Cho IR, Kang H, Jo JH, Lee HS, Chung MJ, Park JY, et al. Efficacy and treatment-related adverse events of gemcitabine plus nab-paclitaxel for treatment of metastatic pancreatic cancer "in a Korean" population: a single-center cohort study. Semin Oncol. 2017:44:420-7.

32. Ko AH, Murphy PB, Peyton JD, Shipley DL, Al-Hazzouri A, Rodriguez FA, et al. A randomized, double-blinded, phase II trial of gemcitabine and nab-paclitaxel plus apatorsen or placebo in patients with metastatic pancreatic cancer: the RAINIER trial. Oncologist. 2017;22:1427-e129.

33. Xu R, Yu X, Hao J, Wang L, Pan H, Han G, et al. Efficacy and safety of weekly nab-paclitaxel plus gemcitabine in Chinese patients with metastatic adenocarcinoma of the pancreas: a phase II study. BMC Cancer. 2017:17:885.

34. Kang J, Hwang I, Yoo C, Kim KP, Jeong JH, Chang HM, et al. Nab-paclitaxel plus gemcitabine versus FOLFIRINOX as the first-line chemotherapy for patients with metastatic pancreatic cancer: retrospective analysis. Invest New Drugs. 2018;36:732-41.

35. You MS, Ryu JK, Choi YH, Choi JH, Huh G, PaikWH, et al. Efficacy of nab-paclitaxel plus gemcitabine and prognostic value of peripheral neuropathy in patients with metastatic pancreatic cancer. Gut Liver. 2018;12:728-35.

36. Boone BA, Steve J, Krasinskas AM, Zureikat AH, Lembersky BC, Gibson MK, et al. Outcomes with FOLFIRINOX for borderline resectable and locally unresectable pancreatic cancer. J Surg Oncol. 2013;108:236-41.

37. Ottaiano A, Capozzi M, Divitiis CDE, Arx CV, Girolamo EDI, Nasti G, et al. Nab-paclitaxel and gemcitabine in advanced pancreatic cancer: the oneyear experience of the National Cancer Institute of Naples. Anticancer Res. 2017:37:1975-8.

38. Loprinzi CL, Reeves BN, Dakhil SR, Sloan JA, Wolf SL, Burger KN, et al. Natural history of paclitaxel-associated acute pain syndrome: prospective cohort study NCCTG N08C1. J Clin Oncol. 2011;29:1472-8.

39. Reeves BN, Dakhil SR, Sloan JA, Wolf SL, Burger KN, Kamal A, et al. Further data supporting that paclitaxel-associated acute pain syndrome is 
associated with development of peripheral neuropathy: North Central Cancer Treatment Group trial N08C1. Cancer. 2012;118:5171-8.

40. Pachman DR, Qin R, Seisler DK, Smith EM, Beutler AS, Ta LE, et al. Clinical course of oxaliplatin-induced neuropathy: results from the randomized phase III trial N08CB (alliance). J Clin Oncol. 2015;33:3416-22.

41. Smith EM, Pang H, Cirrincione C, Fleishman S, Paskett ED, Ahles T, et al. Effect of duloxetine on pain, function, and quality of life among patients with chemotherapy-induced painful peripheral neuropathy: a randomized clinical trial. JAMA. 2013:309:1359-67.

42. Avan R, Janbabaei G, Hendouei N, Alipour A, Borhani S, Tabrizi N, et al. The effect of pregabalin and duloxetine treatment on quality of life of breast cancer patients with taxane-induced sensory neuropathy: a randomized clinical trial. J Res Med Sci. 2018;23:52.

43. Salehifar E, Janbabaei G, Hendouei N, Alipour A, Tabrizi N, Avan R. Comparison of the efficacy and safety of pregabalin and duloxetine in taxane-induced sensory neuropathy: a randomized controlled trial. Clin Drug Investig. 2020:40:249-57.

44. Baba M, Matsui N, Kuroha M, Wasaki Y, Ohwada S. Mirogabalin for the treatment of diabetic peripheral neuropathic pain: a randomized, double-blind, placebo-controlled phase III study in Asian patients. J Diabetes Investig. 2019;10:1299-306.

45. Loprinzi CL, Lacchetti C, Bleeker J, Cavaletti G, Chauhan C, Hertz DL, et al. Prevention and management of chemotherapy-induced peripheral neuropathy in survivors of adult cancers: ASCO guideline update. J Clin Oncol. 2020;38:3325-48.

46. Brierley JD, Gospodarowicz MK, Wittekind C. TNM-classification of malignant tumours. 8th ed. New Jersey, NJ: Wiley-Blackwell; 2017.

47. Pusceddu S, Ghidini M, Torchio M, Corti F, Tomasello G, Niger M, et al. Comparative effectiveness of gemcitabine plus nab-paclitaxel and FOLFIRINOX in the first-line setting of metastatic pancreatic cancer: a systematic review and meta-analysis. Cancers (Basel). 2019:11:484.

48. Ishibashi K, Okada N, Miyazaki T, Sano M, Ishida H. Effect of calcium and magnesium on neurotoxicity and blood platinum concentrations in patients receiving mFOLFOX6 therapy: a prospective randomized study. Int J Clin Oncol. 2010;15:82-7.

49. Loprinzi CL, Qin R, Dakhil SR, Fehrenbacher L, Flynn KA, Atherton P, et al. Phase III randomized, placebo-controlled, double-blind study of intravenous calcium and magnesium to prevent oxaliplatin-induced sensory neurotoxicity (N08CB/Alliance). J Clin Oncol. 2014;32:997-1005.

50. Wesselink E, Winkels RM, van Baar H, Geijsen A, van Zutphen M, van Halteren HK, et al. Dietary intake of magnesium or calcium and chemotherapy-induced peripheral neuropathy in colorectal cancer patients. Nutrients. 2018;10:398.

51. Nishioka M, Shimada M, Kurita N, Iwata T, Morimoto S, Yoshikawa K, et al. The Kampo medicine, Goshajinkigan, prevents neuropathy in patients treated by FOLFOX regimen. Int J Clin Oncol. 2011;16:322-7.

52. Kaku H, Kumagai S, Onoue H, Takada A, Shoji T, Miura F, et al. Objective evaluation of the alleviating effects of Goshajinkigan on peripheral neuropathy induced by paclitaxel/carboplatin therapy: a multicenter collaborative study. Exp Ther Med. 2012;3:60-5.

53. Kono T, Hata T, Morita S, Munemoto Y, Matsui T, Kojima H, et al. Goshajinkigan oxaliplatin neurotoxicity evaluation (GONE): a phase 2 , multicenter, randomized, double-blind, placebo-controlled trial of goshajinkigan to prevent oxaliplatin-induced neuropathy. Cancer Chemother Pharmacol. 2013;72:1283-90

54. Oki E, Emi Y, Kojima H, Higashijima J, Kato T, Miyake Y, et al. Preventive effect of Goshajinkigan on peripheral neurotoxicity of FOLFOX therapy (GENIUS trial): a placebo-controlled, double-blind, randomized phase III study. Int J Clin Oncol. 2015;20:767-75.

55. Hirayama Y, Ishitani K, Sato Y, Iyama S, Takada K, Murase K, et al. Effect of duloxetine in Japanese patients with chemotherapy-induced peripheral neuropathy: a pilot randomized trial. Int J Clin Oncol. 2015:20:866-71.

56. Wang J, Li Q, Xu B, Zhang T, Chen S, Luo Y. Efficacy and safety of duloxetine in Chinese breast cancer patients with paclitaxel-induced peripheral neuropathy. Chin J Cancer Res. 2017;29:411-8.

57. Shinde SS, Seisler D, Soori G, Atherton PJ, Pachman DR, Lafky J, et al. Can pregabalin prevent paclitaxel-associated neuropathy?--an ACCRU pilot trial. Support Care Cancer. 2016;24:547-53.

58. de Andrade DC, Jacobsen Teixeira M, Galhardoni R, Ferreira KSL, Mileno PB, Scisci N, et al. Pregabalin for the prevention of oxaliplatin-induced painful neuropathy: a randomized, double-blind trial. Oncologist. 2017;22:1154-e105.

59. Hincker A, Frey K, Rao L, Wagner-Johnston N, Ben Abdallah A, Tan B, et al. Somatosensory predictors of response to pregabalin in painful chemotherapy-induced peripheral neuropathy: a randomized, placebocontrolled, crossover study. Pain. 2019;160:1835-46.

60. Rao RD, Michalak JC, Sloan JA, Loprinzi CL, Soori GS, Nikcevich DA, et al. Efficacy of gabapentin in the management of chemotherapy-induced peripheral neuropathy: a phase 3 randomized, double-blind, placebocontrolled, crossover trial (NOOC3). Cancer. 2007;1 10:2110-8.

61. Magnowska M, lżycka N, Kapoła-Czyż J, Romała A, Lorek J, Spaczyński M, et al. Effectiveness of gabapentin pharmacotherapy in chemotherapyinduced peripheral neuropathy. Ginekol Pol. 2018;89:200-4.

62. Aghili M, Zare M, Mousavi N, Ghalehtaki R, Sotoudeh S, Kalaghchi B, et al. Efficacy of gabapentin for the prevention of paclitaxel induced peripheral neuropathy: a randomized placebo controlled clinical trial. Breast J. 2019;25:226-31.

63. Xiao W, Boroujerdi A, Bennett GJ, Luo ZD. Chemotherapy-evoked painful peripheral neuropathy: analgesic effects of gabapentin and effects on expression of the alpha-2-delta type-1 calcium channel subunit. Neuroscience. 2007;144:714-20.

64. Gauchan P, Andoh T, Ikeda K, Fujita M, Sasaki A, Kato A, et al. Mechanical allodynia induced by paclitaxel, oxaliplatin and vincristine: different effectiveness of gabapentin and different expression of voltage-dependent calcium channel alpha(2)delta-1 subunit. Biol Pharm Bull. 2009;32:732-4.

65. Peng $P, X i$ Q, Xia S, Zhuang L, Gui Q, Chen Y, et al. Pregabalin attenuates docetaxel-induced neuropathy in rats. J Huazhong Univ Sci Technolog Med Sci. 2012;32:586-90.

66. Aoki M, Kurauchi Y, Mori A, Nakahara T, Sakamoto K, Ishii K. Comparison of the effects of single doses of elcatonin and pregabalin on oxaliplatin-induced cold and mechanical allodynia in rats. Biol Pharm Bull. 2014;37:322-6.

67. Boroujerdi A, Zeng J, Sharp K, Kim D, Steward O, Luo DZ. Calcium channel alpha-2-delta-1 protein upregulation in dorsal spinal cord mediates spinal cord injury-induced neuropathic pain states. Pain. 2011;152:649-55.

68. Barclay J, Balaguero N, Mione M, Ackerman SL, Letts VA, Brodbeck J, et al. Ducky mouse phenotype of epilepsy and ataxia is associated with mutations in the Cacna2d 2 gene and decreased calcium channel current in cerebellar Purkinje cells. J Neurosci. 2001;21:6095-104.

69. Domon Y, Arakawa N, Inoue T, Matsuda F, Takahashi M, Yamamura N, et al. Binding characteristics and analgesic effects of mirogabalin, a novel ligand for the $a(2) \delta$ subunit of voltage-gated calcium channels. J Pharmacol Exp Ther. 2018;365:573-82.

\section{Publisher's Note}

Springer Nature remains neutral with regard to jurisdictional claims in published maps and institutional affiliations. 